\title{
PARA LLEGAR A LA PROBLEMGESCHICHTE: UNA INTRODUCCIÓN METODOLÓGICA A LA HISTORIA DEL PROBLEMA
}

\author{
ACHIEVING PROBLEMGESCHICHTE: \\ A METHODOLOGICAL INTRODUCTION TO PROBLEM HISTORY
}

\section{Armando Torres Fauaz*}

\section{RESUMEN}

Este artículo analiza el aparato metodológico propuesto por O.G. Oexle, según se encuentra expuesto en sus libros L'historisme en débat: de Nietzsche à Kantorowicz, Krise des Historismus, Krise der Wirklichkeit y Das Problem der Problemgeschichte. Se hará énfasis en el desarrollo de dicho aparato, en sus propuestas y en su aplicabilidad al contexto latinoamericano. El análisis de sus consecuencias para las ciencias sociales y naturales en el contexto de la academia contemporánea son de vital importancia para este artículo.

PALABRAS CLAVE: OTTO GERHARD OEXLE * AMÉRICA LATINA * METODOLOGÍA * CIENCIAS SOCIALES * HISTORICISMO * EPISTEMOLOGÍA

\section{ABSTRACT}

This article analyses the methodological apparatus set forth by O. G. Oexle, in his mainly studies Oexle's works entitled L'historisme en débat: de Nietzsche à Kantorowicz, Krise des Historismus, Krise der Wirklichkeit, and Das Problem der Problemgeschichte. This paper emphasizes the development of Oexle's methodology, its theoretical implications and its applicability in the Latin American context. The consequences of this methodology for the social and natural sciences in contemporary academy remain of vital importance for this paper.

KEYWORDS: OTTO GERHARD OEXLE * LATIN AMERICA * METHODOLOGY * SOCIAL SCIENCES * HISTORICISM * EPISTEMOLOGY 


\section{INTRODUCCIÓN}

Este artículo estudia el marco metodológico propuesto por el historiador y epistemólogo Otto Gerhard Oexle. Su objetivo es profundizar en un análisis práctico de la metodología oexliana en función de su aplicabilidad en el contexto latinoamericano.

El trabajo de O.G. Oexle (Singen am Hohentwiel, Alemania, 1939) resulta invitante no sólo por su lucidez y su carácter innovador, sino por el compromiso explícito del autor con un análisis de la discusión académica en relación con su contexto social e histórico. Oexle, quien ha dedicado su vida a hacer las veces de puente entre las ciencias básicas, la filosofía y la historia, es un académico singular. Especialista en Historia Medieval (Freiburg, 1958), Oexle ha explotado paralelamente, temas de la historia cultural de la Edad Media, la Epistemología y la Historia de la Ciencia a finales del siglo xix y principios del xx. Es en el marco del estudio de estos últimos dos temas, que Oexle articula una propuesta metodológica basada en la epistemología de Karl Mannheim (1924) y Reinhart Koselleck (2006), cuyo objeto es el estudio de los problemas científicos y académicos en relación a un "contexto problemático", el cual, compuesto de un conjunto de relaciones interconectadas y contradictorias, codetermina la solubilidad de los problemas académico-científicos. Oexle denomina a dicha metodología "Problemgeschichte" (la historia del problema).

En las siguientes páginas se realizará un estudio de los principios metodológicos de la Problemgeschichte. Luego, se analizará paso a paso, su puesta en práctica por el mismo Oexle. Finalmente, se sopesará la aplicabilidad de esta metodología en el contexto latinoamericano.

Se partirá del análisis de tres obras escritas por O.G. Oexle. En las dos primeras, se explica los principios metodológicos de la Problemgeschichte, una metodología que Oexle considera distinta a la historia de las ideas, a la filosofía de la ciencia y también a la historia de las mentalidades (Oexle, 2001a y 2001b). En cambio, la tercera obra permite estudiar de cerca un ejemplo de la puesta en práctica de la metodología oexliana. El objetivo de esta es analizar el impacto que tuvo la constelación de ideas alrededor del problema del historicismo en la conceptuación de la verdad, por parte de varias disciplinas científicas durante la época de 1880 y 1933 (Oexle, 2007). Este ejemplo se analizará únicamente con el fin de estudiar en la práctica, la operacionalidad de los principios metodológicos oexlianos. En ese sentido, vale aclarar que no es el interés de este artículo observar el desarrollo de las tendencias "historicista" o positivista en Latinoamérica, como lo hizo Oexle para Europa. Dicho trabajo ha sido realizado lúcidamente, entre otros, por Abraham Moctezuma Franco para el México de 1940 (Moctezuma, 2004).Por otro lado, en este artículo interesa un regreso a la base metodológica de la Problemgeschichte y un examen de su aplicabilidad para la historia de la academia y de la intelectualidad latinoamericanas.

\section{LA FORMULACIÓN DE LA METODOLOGÍA DE LA PROBLEMGESCHICHTE}

Las primeras cavilaciones acerca de una metodología para el estudio de los problemas científicos y epistemológicos en retrospectiva, las realizó Oexle durante sus años de labor en el Max Planck Institut. La articulación de las observaciones de Oexle sobre las tesis historiográficas de Max Weber y de los estudios sobre Ernst Cassirer realizados por Michael Hänel (2001: 85-128), así como, los estudios sobre Karl Mannheim realizados por Reinhard Laube (2004: 27-40 y 59-85), permitió la definición de los principios metodológicos de lo que vendría a recibir el nombre de Problemgeschichte (Oexle, 2001b: 25-32).

La wissenchaftige Problemgeschichte, Oexle la distingue de la historia del pensamiento, la historia de las ideas y la historia de la ciencia. Con el afán de resignificar el término ${ }^{1}$

1 El término alemán "Problemgeschichte" no es nuevo. Oexle se lo reapropia y efectúa un giro semántico que aleja el significado que adquiere el término en su metodología del significado al que más tradicionalmente refiere: a saber, la reconstrucción de la historia de las ideas por medio de una exégesis clásica. Ver el significado que, por ejemplo, toma el término en el volumen Stallmach (1959) o en el libro de Theo Kobusch, Problemgeschichte der Antiken 
(Stallmach, 1959), Oexle opera el concepto de Problemgeschichte como una metodología a través de la cual se busca reconstruir la totalidad del debate alrededor de un problema científico o epistemológico. No se trata de una profundización exegética, se trata de reconstruir la "constelación" de preguntas y respuestas científicas alrededor de un concepto o un grupo de conceptos. La Problemgeschichte da una importancia primordial a la formulación de la pregunta como etapa previa a la constitución de un proyecto epistemológico. No se busca una categorización ni una periodización de las ideas, sino el análisis de un problema-protagonista alrededor del cual se proponen y discuten posibles soluciones desde diversas teorías, puntos de vista y disciplinas (Oexle, 2001b: 9-38). El valor que tienen el análisis histórico-contextual $y$ multidisciplinario en la metodología de Oexle, lo aleja en cierta medida de análisis de tipo más claramente epistémico, como el que T. Viehweg (1953) realiza para el derecho valiéndose de la antinomia Problemdenken-Systemdenken, inicialmente planteada por Hartmann (1957: 281-282) en su crítica a la epistemología kantiana. Sin duda, el rol central de los topói (planteamiento, estructuración y persecución del problema) como motor de la empresa científica y como recurso heurístico para la organización de un sistema, tal como lo plantea Viehweg (1953: §3), trasparece en las premisas de Oexle. No obstante, la importancia del problema, en tanto definitorio de cualquier proyecto cognitivo, llega a Oexle por vía del estudio de la epistemología de Karl Mannheim (1924), como se verá a continuación.

La Problemgeschichte es interdisciplinaria por excelencia. Dado que su objetivo es comprender la totalidad de respuestas o reacciones alrededor de un problema-protagonista, se hace necesario, primero, analizar de qué forma ese problema fue abordado en el seno de una multiplicidad de disciplinas o prácticas científicas, académicas y artísticas, y segundo, examinar de qué forma esas reacciones

und mittelalterlichen Philosophie, Leyden: Brill, 1987 o en los cinco volúmenes de Hans Heinz Holz, Problemgeschichte: Der Antiken bis zum Gegenwart. Darmstadt: WGB, 2010. interactuaron en un escenario común. A este ejercicio, Oexle lo llama la reconstrucción de la Problemskonstellation, es decir, la constelación de preguntas y respuestas alrededor de un problema-protagonista (Oexle, 2001b: 64). Esta primera fase deriva del pensamiento de Karl Mannheim, quien en 1925, había pensado en el problema de la historia de la cultura (articulada más bien, como historia del espíritu) bajo la premisa de substituir el Geist diltheiano (Dilthey, 2006) por un Problembezug (relación de problemas), tal que fuera posible, no solo la presentación de un problema en $-y$ desdesus distintos resultados, sino también el análisis "de la constelación de factores extra-teóricos que codeterminan los posibles pasos hacia su reconocimiento y que contienen su solubilidad [Lösigkeit]" (Mannheim, 1924: 578).

Como premisa metodológica elemental, Oexle plantea una distinción entre las acciones de Re-problematisierung y Ent-problematisierung (Oexle, 2007: 20 y 109). En otros términos, la acción de Ent-problematisierung equivale a hacer más complicado un problema que es ya de por sí complicado; es decir, implica el entorpecimiento de la aclaración o reformulación de un problema, impidiendo que este pueda rearticularse partiendo de una perspectiva exógena. El verbo Ent-problematisieren designa el resultado de un acercamiento no-metódico y poco reposado sobre un problema del pasado. Implica revivir una polémica ataviada de prejuicios sin procurar el análisis crítico de su objeto desde una nueva perspectiva. Según Oexle, la Ent-problematisierung puede pensarse, entonces, como un obstáculo a la Erklärung, un impedimento de la Aufhebung.

En cambio, la Re-problematisierung es el proceso por medio del cual se le da un nuevo soplo al tratamiento de un problema antiguo. Se trata de replantear el problema, tal que pueda articulársele en una discusión actual, habiendo de antemano subrayado la validez de su objeto $y$ la pertinencia de su retrotracción al centro del debate académico. Para insistir sobre Mannheim, puede afirmarse que la $R e$ problematisierung, la cual constituye la labor esencial de la Problemgeschichte, es entonces 
una re-concienciación del problema, un Probl embewußtseinwerden (Laube, 2004: 45-47 y 59).

Dado que la Problemgeschichte en efecto procede y se nutre de la metodología de la historia, el factor del tiempo es igualmente central para el análisis. No se trata de petrificar la discusión alrededor del problema protagonista, sino de dinamizarla sincrónica y diacrónicamente. El estudio de la Problemkonstellation se hace, entonces, en el tiempo, observando y analizando las distintas respuestas y direcciones que toma la discusión en un lapso determinado. Lapso que, por lo demás, no busca reproducir tendencias marcadas por la historia de las ideas, sino que es más la consecuencia de una periodización anclada en lo social. Por consiguiente, aún si el protagonista es el problema, Oexle es enfático al subrayar la necesidad de contextualizar la Problemkonstellation. Contextualizar, además, no es otra cosa que circunscribir la Problemkonstellation a las condiciones sociales en las que fue tejida (Oexle, 2001b: 75-78 y 128-131). Esa premisa metodológica es precisamente lo que diferencia a la Problemgeschichte de la llamada Historia de las Ideas, anclada la mayoría de las veces, en una simple sucesión de teorías reductibles o en un 'biografismo’ de sus más reputados agentes.

Por demás, dado que el objeto de la Problemgeschichte es el análisis de un problemaprotagonista definido dentro de la esfera de lo intelectual, esta metodología no puede asimilarse a la Historia de las Mentalidades, la cual se ocupa, es bien sabido, de un análisis etnográfico del pensamiento colectivo, sus ideas, sus manifestaciones y sus representaciones (Ariès, 1978: 167 y 177-182). En este marco correlacional negativo, la Problemgeschichte puede describirse mejor como el análisis de la efectuación de una transformación paradigmática, tal como la entendería Thomas Kuhn (1996: 43-51), así como de las condiciones sociales en que esta se efectúa.

Ahora bien, en la práctica, Oexle da un giro aún más interesante en el desarrollo de la Problemgeschichte. Al realizar un estudio sobre el problema del historicismo, utilizando la metodología planteada, Oexle (2007) se revierte al origen de las ideas que gobiernan los conceptos de caducidad, sustitución, temporalidad, falsación, etc., los cuales se ubican en el centro del debate epistemológico alrededor de la Verdad. En otros términos, Oexle somete a un análisis de Problemgeschichte, al precepto que le da coherencia a esta metodología, a saber: la idea de que la verdad es historizable.

Con el fin de comprender la efectuación práctica de la metodología de la Problemgeschichte, se procederá a analizar en detalle cómo Oexle pone en práctica la metodología que plantea. No solo porque así pueda dilucidarse otro tipo de problemática intrínseca a la práctica, sino porque solo por medio del análisis práctico de la Problemgeschichte, es posible pensar su aplicabilidad en el contexto latinoamericano.

\section{LA PROBLEMGESCHICHTE PUESTA EN PRÁCTICA}

En su obra, Krise des Historismus, Krise der Wirklichkeit, Oexle (2003) analiza el problema del historicismo como una forma de percibir el mundo $y$ de producir conocimiento.

Apegándose a la metodología de la Problemgeschichte, el estudio de Oexle se divide en varias etapas. En la primera, comienza por identificar el problema-protagonista, a saber: el historicismo. Procede a continuación a exponer las ideas propuestas por los defensores de esta perspectiva, con el fin de determinar el concepto $y$ articular el problema. Luego, procede la reconstrucción de la Problemkonstellation. Con este fin, estudia las ideas de los opositores del historicismo. Producto de esta primera fase del análisis, Oexle logra determinar que la Problemkonstellation alrededor del historicismo, ubica a este último en una relación de contraposición con respecto al positivismo y a la noción científica y académica de una verdad única, espiritual (geistliche) $y$ aprehensible.

En la segunda fase de la investigación, Oexle procede a estudiar otras manifestaciones colectivas relacionadas a la idea de perennidad/ caducidad, totalidad/relatividad, tales como: el derecho, la economía, la plástica, la filosofía y la literatura, con el fin de procurar la "contextualización problemática" de la Konstellation. Determina, que durante el período estudiado, 
el problema del historicismo no estaba únicamente confinado a la ciencia o a la epistemología, sino que la constelación de preguntas $y$ respuestas que le concernían, rebasaba los límites de la academia, para tocar no solo la esfera de la "alta cultura", sino también otras sensibilidades más extensas y generales, que podían acercarse más a nociones colectivas socialmente transversales.

En una tercera etapa, Oexle historiza la Problemkonstellation. El grueso de la investigación está dedicado a esta operación, que consiste en el estudio retrospectivo de la constelación alrededor del problema-protagonista, realizado tanto sincrónica como diacrónicamente. En consecuencia con los principios metodológicos de la Problemgeschichte, el análisis de Oexle implica además una puesta-enrelación de la Problemkonstellation con las condiciones histórico-sociales en que surgen las distintas controversias.

Al final de este proceso, el autor logra establecer una relación plausible entre lo que él llama 'la crisis del historicismo' y 'la crisis de la Realidad/Verdad', demostrando por una vía alternativa a la exégesis, que existe una relación histórico-epistemológica entre los conceptos de caducidad $y$ relacionalidad, $y$ los de verdad $y$ totalidad. Amparado en la sociología relacional de Simmel (1992d: 456-511), Oexle ubica además esta relación en un contexto social dinámico, el cual pasa por varias etapas. La primera, caracterizada por el convencimiento general acerca del carácter aprehensible de la realidad (1850-1880). La segunda, inundada de dudas con respecto a la certeza del conocimiento $y$ al sentido de la historia (1880- ca. 1933). La última, dominada por el totalitarismo (ca. 1933-1945).

Dicha empresa constituye, en fin, un ejercicio de reproblematización (Re-problematisierung) del problema-protagonista, es decir, una revalorización del historicismo como tema para las ciencias sociales $y$ la retrotracción de este al centro de la discusión académica. En las páginas siguientes, se estudiará paso a paso la puesta en práctica de la metodología oexliana, con el fin de "diseccionar", si se quiere, la obra práctica de Oexle.
PRIMER PASO: IDENTIFICACIÓN Y ESTUDIO DEL PROBLEMA-PROTAGONISTA

\section{LA KRISE DES HISTORISMUS}

El autor parte de una premisa: el historicismo es la idea fundante de la Modernidad $y$ de lo moderno. El término se refiere a la conciencia de la temporalidad de lo existente y de lo conocido, extendiéndose incluso, a la conciencia sobre la caducidad del ego. La primera y más importante definición del historicismo la toma Oexle de Ernst Troeltsch, quien en 1922 lo definía como: "la historización de todos nuestros conocimientos y descubrimientos acerca del mundo del espíritu (geistigen Welt), así como fue concebido a lo largo del siglo xix" (Troeltsch, 1922: 14). Esta definición no es por demás discordante con el proyecto que emprende Dilthey, de escribir una historia del espíritu (Geist) para el siglo xix (Dilthey, 2006).

El historicismo del siglo xix, a pesar de no haber procurado una autodenominación, según Oexle, tuvo un efecto semejante sobre la Wirklichkeit (Realidad/Verdad) al que sobre ella tendría el historicismo del siglo xx. Nietzsche, en 1874 , se oponía a la validez de la historia como método de explicación de lo real, debido a la insistencia de esta en estudiar el hecho particular del pasado y procurar su transmisión al presente como una verdad/razón determinante de lo contemporáneo. Juzgaba la historia como una enfermedad de la modernidad, esclava de una moral conservadora, un encadenamiento del presente al pasado $y$ por consiguiente, el más claro impedimento de la liberación de la esencia humana verdadera. Sin embargo, habrá que esperar al artículo de Troeltsch para obtener una crítica consciente acerca de la 'crisis del historicismo', que por lo demás, generó una discusión epistemológica harto importante. Para Troeltsch, esta crisis tenía tres dimensiones:

A) El cuestionamiento de la objetividad del conocimiento concebido como la relación entre el pensamiento (el orden de los objetos pensados) y la disposición real de los objetos (la esencia de la cosa misma).

B) La unidimensionalización de las verdades frente a la historización de las convenciones. 
C) El problema ético de un relativismo moral y de los valores que iban ligados al análisis crítico de las condiciones de posibilidad del conocimiento.

\section{DIE KRISE DER WIRKLICHKEIT}

El término utilizado por Oexle, Krise der Wirklichkeit, nos compele al intento de aclarar el concepto detrás de la voz alemana Wirklichkeit. Anclados en la tradición idealista alemana, se podría considerar que la Wirklichkeit, más que simple "realidad" (Realität) o manifestación tangible, es no solo en tanto forma de lo esencial, sino como única manifestación de lo inteligible. En otros términos, es aquello sobre lo que recae el juicio de lo verdadero (Hegel, 1988, §16-23). Así, cuando la Wirklichkeit entra en crisis, para Oexle, no solo entra en crisis la realidad tangible, sino la efectividad que la acciona ${ }^{2}$ (Hyppolite, 1967: 24), dígase todo aquello que puede manifestar lo verdadero para quien la juzga. Una crisis de la Wirklichkeit es entonces, para la ciencia, una crisis de la Realidad/Verdad. Esto, pues los juicios que la ciencia emita a partir de una Wirklichkeit que no reconoce como manifestación inteligible del principio universal, no pueden ser efectivos intersubjetivamente. Puesto de otra forma, ninguna ley científica es lógicamente necesaria ni universalmente aplicable, si la totalidad y la verdad absoluta de su identificación, en tanto totalidad, no se consideran aprehensibles mediante el sistema de la ciencia.

En Oexle, esta Wirklichkeit toma dos formas. Primero, la forma de una realidad natural que sostiene $y$ acciona la realidad manifiesta, además de imprimirle lógica a los fenómenos del mundo objetivo. La convicción de la efectividad de esta realidad natural superior entra en crisis cuando la física y la química comienzan a dudar de su aprehensibilidad. Segundo, la forma de una totalidad inteligible sobre la que recae una verdad absoluta a la que se sintonizan los descubrimientos de la raza humana. La convicción de la aprehensibilidad de esta verdad entra en crisis cuando el historicismo niega sus condiciones de posibilidad, al afirmar la temporalidad, la falsabilidad y la caducidad de las acciones y resoluciones humanas.

Así, la Krise der Wirklichkeit se refiere a un proceso de doble lógica, una cabeza de Janus, para ponerlo en términos de Koselleck (1987: 27). Primero, los descubrimientos de Heisenberg, Bohr, Einstein y Planck presentaron una realidad física dinámica, relativa y dependiente de su observador, negando así la existencia de una Realidad/Verdad acabada, aprehensible y externa al sujeto. Luego, las proposiciones del historicismo negaban la Realidad/Verdad en su manifestación absoluta $y$ totalizante. Por una parte, los logros y verdades humanas aparecían temporales $y$ contingentes - entiéndase, superables. Por otra parte, algunos defensores del historicismo llegaron a comparar la empresa científica-natural con la historiográfica, considerando que ambas construían - no descubrían - la realidad que luego asumían como verdadera. Así, la Verdad resultaba enteramente dependiente del método $y$ de las presuposiciones contextuales o axiomáticas que motivaban su afirmación.

Debido a esta "crisis", la Realidad/Verdad (la Wirklichkeit) y su carácter esencialmente aprehensible, dejaban de ser una certeza incontestable. Según Oexle, la 'crisis de la Realidad/ Verdad' fue concebida como concepto y problema en 1929, con el artículo de Ludwig Fleck. Motivado por los descubrimientos ligados a la teoría de los quanta de Einstein y Planck, además del problema del Principio de Indeterminabilidad de Heisenberg y el de la Complementariedad de Bohr, Fleck concluía que la observación y el conocimiento son siempre una puesta-en-relación (Beziehungseingehen), una transformación del objeto del saber; he ahí la labor cotidiana de la ciencia. Para Fleck resultaba evidente que el objeto y el sujeto no tienen una Realidad independiente el uno del otro; consecuentemente, el conocer - aprehenderno se trata de una afección involuntaria sufrida por una tabula rasa, ni de una acción unilateral del sujeto sobre lo que percibe con los sentidos: "conocer es más una cotidiana y constante puesta-en-relación, un transformar y ser transformado, en breve: un crear" (Oexle, 2003: 15) ${ }^{3}$.

$3 \quad$ En el original: "Erkennen sei vielmehr ein tätiges, lebendiges Beziehungseingehen, ein Umformen und Umgeformtwerden, kurz ein Schaffen".

2 Cfr. Introducción. En especial la nota 24, p. 135. 
SEGUNDO PASO: LA RECONSTRUCCIÓN DE LA PROBLEMKONSTELLATION

\section{LA RELACIÓN ENTRE LAS DOS CRISIS}

Oexle se remonta a los orígenes del historicismo europeo en la segunda mitad del siglo xvIII, con el fin de estudiar la problemática alrededor del historicismo y la relación entre la crisis del historicismo y la crisis de la Realidad/ Verdad en la forma en la que se desarrolló entre 1880 y 1933, en Alemania. Ahí encuentra dos puntos de partida para el historicismo como explicación de lo real. El primero está en el pensamiento epistemológico de Kant, especialmente, en el tratamiento del ideal de la razón práctica que engendra la pregunta sobre si la humanidad está siempre en constante progreso hacia lo mejor (1798). El segundo es el descubrimiento científico de la Edad Media, que permitió por primera vez, la distinción y contraste entre lo presente - lo Moderno-y lo pasado -lo Antiguo (Oexle, 2003: 25).

Oexle explica cómo en el siglo xviI, el Medioevo se constituyó en el concepto (Begriff) de período por excelencia (Oexle, 2003: 27-34). El descubrimiento de la Edad Media fue el primer paso hacia el descubrimiento de lo histórico an sich, pues permitió la inauguración de una conciencia académica acerca del pasado e impuso una concepción de la historia de la humanidad como una sucesión lógica de períodos históricos (eras). Así, el descubrimiento de la Edad Media y el establecimiento de una diferencia clara entre lo medieval y lo moderno comportaban una idea de movimiento, de cambio de época, ergo, de caducidad. La conciencia de una diferencia de épocas implicaba la conciencia de la finitud del presente. Oexle concuerda entonces con Koselleck cuando afirma que la idea del historicismo nace entonces al mismo tiempo que $-y$ estrechamente ligada a- la idea de progreso: "la concepción histórica y la concepción progresista del mundo y tienen un mismo origen y se complementan la una a la otra como el rostro de Ianus" (Koselleck, 1987: 27).

Así, el historicismo es constitutivo y específico de la modernidad, pues es la historización comprehensiva del mundo, de las gentes $y$ de todas las dimensiones de la cultura. Como tal, en efecto, el historicismo se contrapone a la idea de una realidad inmutable y absoluta, aún si esa realidad es el resultado del estudio de la historia. Todo está en movimiento, todo tiene final.

Para Oexle, consecuentemente, fue entre los historiadores y no entre los científicos naturales, que comenzó a cuestionarse la Realidad/ Verdad que sostenía o caracterizaba los descubrimientos historiográficos, aportando así una constelación de discusiones alrededor de las posibilidades del conocimiento científico.

La exposición de Oexle comienza evidentemente con la figura de Leopold von Ranke, considerado el 'profesionalizador' de la historia como disciplina. Sin embargo, Oexle no presenta a Ranke como el primer historiador positivista o el positivista por excelencia reputación que lo ha perseguido durante más de un siglo- sino como un sacerdote de la Religión de la Historia. Oexle consideraba que Ranke, quien deseaba exponer las cosas "tal y como habían sido" (wie sie eigentlich gewesen), esbozaba el proceso del conocimiento histórico casi como una epifanía, pues esperaba encontrar la Verdad tal cual en los documentos, carente de toda mediación, transformándose el historiador casi en un canal entre los acontecimientos pasados y el hoy, desde donde se estudian: "deseaba igualmente suprimirme y narrar sólo las cosas tal cuales, dejar evidenciarse a las fuerzas poderosas" (von Ranke, 1905, citado por Oexle, 2003: 54).

En el sentido contrario de esta anulación del sujeto, fue Johan Gustav Droysen, en 1887, el primero que propusiera la empresa historiográfica como un proceso empírico cuyos resultados eran construidos intelectualmente. Según Droysen, la metodología historiográfica estaba opuesta a la metodología de las ciencias naturales, de tal forma que la segunda creía en una Realidad por descubrir, mientras la primera reconocía el resultado de su empresa como un constructo y no como la descripción de una realidad exterior al sujeto: “¿entonces cuál es la Realidad/Verdad del conocimiento histórico? Es, como dijo Droysen, no una fotografía del pasado, sino un esbozo, una representación de la realidad de ese pasado" (Oexle, 2003: 55). 
Las ciencias naturales parten de una realidad física y material tangible y aprehensible. Para Droysen, el único elemento físico y tangible de la historia son los documentos o lo que él llamaba el "material histórico"; en sí misma la realidad histórica (como totalidad) permanecía siendo abstracta. De esta forma, Droysen se oponía a un tiempo a la existencia de una Realidad fuera del observador $y$ a las pretensiones de una historiografía rankeana de 'lo que en verdad pasó'.

Una posición similar fue defendida por Georg Simmel, en 1896. Sin embargo, la oposición entre la historia y las ciencias establecidas (gesetzte Wissenschaften) era demasiado fuerte para Simmel; tanto que la oposición se vio inundada de juicios de valor. Según Simmel, Kant significaba para la sociedad de fines del siglo xix, no solo una limitación de las posibilidades de nuestro conocimiento, sino también la afirmación de que el mundo conocido no era extraño a la estructura de nuestra conciencia. Si se seguía a Kant, se andaba por un camino de enriquecimiento infinito, a lo largo del cual se descubría la forma constitutiva de nuestro conocimiento. Aprehendiendo el mundo sensible, nos conocíamos a nosotros mismos. De esa forma, el valor principal para la Verdad es según Simmel (1992c: 349) la relación (Beziehung). Si se busca saber lo que verdaderamente fue, se encontrará una serie infinita de preguntas que surgirá después de cada respuesta, pues cada conocimiento nuevo engendra por medio de la razón, nuevas interrogantes. La Verdad/Realidad única es la "relacionalidad" y la acción de relacionar es el único método de aprehensión de lo Real/Verdadero.

Las ciencias naturales se equivocaban. Buscaban estudiar los fenómenos del mundo de una forma estable y no encontraban sino formaciones físicas, éticas y sociales afectadas por un desarrollo constante, en las cuales cada elemento estaba solamente limitado por su relación con su antes y con su después. La historiografía era la única que explicaba la realidad, a partir de una red de interdependencias complejas (Simmel, 1992c: 764-771). De ahí que la disciplina de la historia
(Geschichtwissenschaft) fuera la disciplina de la Realidad/Verdad (Wirklichkeitswissenschaft) por excelencia.

Es de esperar que ante esta noción de Realidad/Verdad, opuesta a la unidimensionalidad positiva que sostenía el viejo método newtoniano, las respuestas de la comunidad científica fueran hostiles. Lo interesante de observar, según lo subraya Oexle, es que dentro de la comunidad de científicos naturales, tan aparentemente convencida de la existencia de una Verdad objetiva del mundo, surgieran interrogantes acerca del proceso deductivo que llevaba al descubrimiento de esa Realidad/Verdad que llevaba el nombre de "científica".

Para las ciencias naturales, fue Niels Bohr, en 1927 — fecha en que se publica el artículo de Fleck- quien primero hizo pública su percepción de que la tarea de la Física ya no era el conocimiento y la organización del mundo, sino la de descubrir lo que podía ser conocido por la ciencia (Bohr, 1984). El mismo año, Werner Heisenberg afirmaba que no había una realidad objetiva independiente de la observación del sujeto (1927: 195-197), las circunstancias de cada observación cambiaban la realidad del objeto observado. Era posible observar al objeto de formas $y$ desde posiciones infinitamente distintas $y$ adjudicarle equivalente número de realidades o verdades características. Así, era imposible aprehender la verdadera naturaleza de un objeto $y$ proporcionar una descripción absoluta y universal del mismo, lo que obligaba a los científicos naturales a darse otra noción de cientificidad que asumiera la imposibilidad de una Realidad/Verdad universal sin negar la alta exactitud que implicaba el concepto de cientificidad. Oexle lo entiende así: "la realidad de esta imposibilidad no negaba la existencia de un conocimiento exacto, devenía más bien una caracterización del conocimiento exacto mismo" (Oexle, 2003: 83).

A su vez, la filosofía de la ciencia se dio también a la revisión de la noción científica positiva de la Realidad/Verdad. Ernst Cassirer y Karl Mannheim propusieron las tesis relacionistas que se oponían a la existencia de una única Realidad/Verdad aprehensible y totalizante. Para Cassirer (1906), los resultados 
de la empresa científica no eran una descripción de lo dado, sino una construcción crítica aprehensible, solo si se le extirpaba su posible variabilidad, inherente a todos los objetos de la observación.

Para Mannheim (1924) era precisamente la "diferencia", la que permitía el análisis de la Realidad/Verdad, pues la diferencia es la relación entre los elementos que constituyen una totalidad aparente. El ser no existe como una unidad indivisible; la Realidad/Verdad total y acabada es una ilusión, una falsa Seinsverbundenheit (unidad de una totalidad) que no constituye sino un primer paso hacia la aprehensión y evidencia de la Seinsgebundenheit (condición de unión de las partes constitutivas de una aparente totalidad) como la verdadera naturaleza de lo que se manifiesta en el mundo. El conocimiento no puede obtenerse sino a partir del análisis relacional entre lo que constituye al ser $y$ lo que le permite manifestarse como una totalidad en el mundo.

Esta nueva perspectiva ya no le permitía a las ciencias naturales actuar con la autoridad platónica de ser el método único y exclusivo de acceso a la Verdad y de explicación de la Realidad. El relacionismo hacía de la Verdad científica una construcción social y el historicismo la ponía en una perspectiva temporal que la transformaba en algo enteramente contingente, finito.

Oexle parece convencido de que fue el historicismo, en tanto tendencia intelectual de la modernidad — es más, como idea fundante de lo moderno - lo que generó una conciencia académica acerca de la finitud de los logros de la humanidad, por consiguiente, acerca de lo contingente de las verdades científicas y la imposibilidad de llegar a afirmar una Ley universal absolutamente verdadera por la vía de la ciencia. Esa concepción y la metodología que en ella se basaba, supone Oexle, era un elemento pseudoconsciente en lo miembros de las academias europeas entre finales del siglo xIx y principios del xx, lo que facilitó la articulación de descubrimientos como los de Heisenberg y Bohr, o los de Planck y Einstein, en una verdadera preocupación intelectual acerca de la imposibilidad del conocimiento de lo verdadero (2003: 47-51).
TERCER PASO: ANÁLISIS DE LA DINÁMICA DE LA PROBLEMKONSTELLATION EN UN CUADRO TEMPORAL

\section{LA REACCIÓN}

Luego de estudiar las respuestas alrededor de la idea del historicismo y de la crisis que genera en la concepción de la Realidad/Verdad para los científicos, lo cual en su investigación constituye la Problemkonstellation, Oexle se da a la tarea de analizar la reacción conservadora de los sectores que no se plegaron a las ideas relacionistas o historistas que surgían.

Según lo explica Oexle, una de las formas más evidentes de negar las proposiciones del historicismo y de reafirmar una Realidad/Verdad única, fue el libro de Friedrich Meinecke, $E l$ desarrollo del historicismo (1936), el cual reducía el historicismo a una forma de pensar banal y superada, característica del idealismo alemán. Meinecke identificaba el historicismo esencialmente con Vico, Humboldt y Herder, además de considerar a Ranke como su representante más característico. Esta forma de desvirtuar al historicismo como una ideología añeja y adjudicada a personajes que le eran esencialmente ajenos, no era solo atractiva para los cientificistas positivos, detractores de esta forma de pensar, sino también para la Intelligentsia de un Estado que estaba en plena exaltación nacionalista y necesitaba de una Verdad innegable: la propia.

Por otra parte, el rechazo de las ideas relacionistas estuvo acompañada de denuestos contra sus portavoces, quienes fueron acusados de profesar un relativismo moral y epistemológico. De esta forma, los relacionistas devinieron nihilistas. La inconsistencia de la totalidad del ser en Mannheim devino un relativismo; así como, las proposiciones de Bohr, Heisenberg, Planck y Cassirer. En este frenesí, explica Oexle (2003: 96), incluso las ideas de Max Weber fueron presentadas como libertinaje $y$ una negación de la moral religiosa. La hostilidad contra el neokantismo como una forma de relativismo, hostil a la metafísica, provocó un fácil rechazo de las teorías de Simmel y Droysen sobre las que triunfaron las proposiciones de Helmolz y Wirschow, para quienes la ciencia positiva y su método constituían la única forma de llegar a la Verdad. Lo que debía buscarse era la unificación 
de las metodologías de todas las disciplinas científicas bajo el ala del método newtoniano que regía a la Física y la Química.

Según describe Oexle, a las proposiciones del relacionismo científico no se opusieron solo las afirmaciones de un positivismo añejo, sino también la de un brutal pragmatismo político. Desde la academia hasta la comunidad científica oficial del Estado y la empresa privada, se procuró desvirtuar todas las ideas que negaran una Realidad/Verdad unidimensional e implacable. Así, cuando el nacional-socialismo ocupó la mayoría de sillas en el congreso alemán y se acabó por elegir a Hitler como Canciller plenipotenciario, la Realidad/Verdad del progreso, del orgullo nacional y de la guerra aplastó cualquier asomo crítico y propulsor de lo factible frente a lo fáctico. Los fascismos germano y latino impusieron una Wirklichkeit ineludible: la brutalidad.

\section{EL ESTANCAMIENTO}

Dada la naturaleza del fascismo, resulta evidente que el régimen alemán nazi procurara una Wirklichkeit única, monolítica y eterna; dígase: una Verdad Totalizante. Así, la ciencia alemana debía siempre llevar a la verdad científica, debía basarse en la Realidad y conducir a su revelación, a su aprehensión y en última instancia, a su dominación e instrumentalización:

La física alemana, debía ser la física aria, la física de las educadas gentes del norte [...] la física de los descifradores de la Realidad, de los buscadores de la Verdad [...]. El deseo de los investigadores alemanes debía ser el desciframiento de la Verdad, el estudio real de la naturaleza; la ciencia alemana debía basarse en la observación, no en la articulación de los fenómenos naturales en un constructo epistémico abstracto $y$ arbitrario (Oexle, 2003: 106).

La verdad que aportaba la ciencia aria era la Verdad de la Vida, tanto la Verdad de la Historia como la Verdad de la Naturaleza. Volver a fundar la sociedad implicaba el asesinato de lo moderno; así, la idea de temporalidad pasaba de ser la conciencia de un fin ineludible a ser una de las características intrínsecas de lo humano. Ein Reich für Tausend Jahre. En la lógica brutalmente utilitarista del fascismo, la certeza de la empresa científica se reducía a su eficacia $y$ aplicabilidad en la guerra. Igualmente, la certeza del desarrollo histórico se materializaba en la fundación del nuevo hombre: el hombre fascista. Podría decirse que la verdad de la ciencia era entonces la victoria (sieg Heil!) y la verdad de la historia, el nazismo.

De esta forma, según Oexle, el debate alrededor del problema del historicismo y de la aprehensibilidad de la Realidad/Verdad; es decir, las llamadas "dos crisis", fue violentamente cortado por el nazismo y por la Segunda Guerra Mundial. No fue sino hasta después de los años 50 , que la discusión fue retomada, sin embargo, carente de la misma claridad y continuidad que había tenido entre 1880 y 1933, pues se ubicaba ya en otro contexto intelectual e incluso nacional/cultural (consecuencia de las migraciones masivas al continente americano).

Para Oexle, el estancamiento del debate no es solo el producto de la persecución de la diferencia o de la afirmación sistemática de la Verdad totalizante, apoyada por el orden nacional-socialista. Un nuevo contexto donde el historicismo y el cuestionamiento de la Realidad/ Verdad ya no tenían cabida comenzaba a manifestarse también desde la década que siguió a la Primera Guerra Mundial, en esferas como el arte (el futurismo italiano, el íncipit del realismo heroico), en la academia (podría subrayarse el caso del matemático Georg Cantor, enloquecido por el rechazo a sus teorías sobre el infinito) y en las mismas ciencias humanas (el medievalismo de Ernst Kantorowicz (1927) y Otto Brunner (1939), y el darwinismo de L. Strauss). Al mismo tiempo, el paso a un mundo de aceleración, de consolidación nacional, de guerra $y$ de transformación constante, traían a la Alemania de principios del siglo xx, una tarea de redefinición, en la cual, es bien conocido, primaron las fuerzas corporativistas y militaristas, las cuales constituyeron una estructura de apoyo oficial a las ideas conservadoras que se oponían al cuestionamiento de la formulación positiva del mundo. 


\section{CONSIDERACIONES: LA APLICABILIDAD DE LA PROBLEMGESCHICHTE}

La academia contemporánea podría caracterizarse, entre otras cosas, por una auténtica pretensión de interdisciplinariedad. Motivado por la nueva urdimbre pluri-, multie interdisciplinaria de algunas ramas de las ciencias básicas, el deseo de un laxamiento de los márgenes entre las disciplinas y de una combinación (reposada, repentina o en ocasiones, oportunista) de las áreas académicas, no ha cesado de avizorar a la academia contemporánea. En esta perspectiva, la proposición de Oexle cumple a cabalidad con dichas expectativas, al tiempo que cuestiona la base misma del edificio académico-científico. Sirviéndose de una efectuación de la noción de interdisciplinaridad, Oexle otorga una serie de instrumentos para comprender que las pretensiones académicas y las "verdades" científicas guardan una relación estrecha con la realidad social en que son concebidas. Este autor no pretende, como algunos marxistas vulgares -más superados que actuales - reducir la "superestructura" a la "infraestructura", sino en cambio, entender que la direccionalidad entre la producción científica, cultural, artística y su contexto social, no es lineal ni unilateral. Al contrario, tal y como lo plantean Simmel (1992), incluso Deleuze (1953) o hasta Zizek (2006), la relación entre los objetos producidos culturalmente, entre las ideas y concepciones académicas y científicas, así como, sus circunstancias sociales, materiales y políticas, es multidireccional, dialógica $y$ enteramente relacional.

Sería incorrecto por demás considerar que la proposición de Oexle se encuentra aislada o que surge ex nihilo. Tal aseveración contradiría los principios más elementales de la metodología oexliana. En ese sentido, no pretende en ningún momento desvincularse por completo de una tradición, de la cual se reconoce heredero; aún si dicha tradición ha sido confinada a una cierta oscuridad. Como antecedentes a su propia metodología, este autor reconoce la visión relacional entre observadores y objeto observado de G. Simmel (1992), la crítica de los conceptos más básicos de la ciencia y de la posibilidad del conocimiento emprendida por K. Mannheim (1924), M. Weber (1904) y E. Cassirer (1906); así como, la reconstrucción crítica de la historia del concepto (Begriffsgeschichte) propuesta por R. Koselleck (2006).

Además de establecer este marco germánico, sería quizá importante reconocer que otros autores, como B. Latour (1979), S. Wooglar (1988) y P. Bourdieu (2001), entre otros, han estudiado a profundidad las circunstancias sociales en que se construye el conocimiento $y$ se establecen las verdades científicas. No obstante, la metodología oexliana aporta una visión que se distingue del examen microscópico y etnográfico, en tanto busca reconstituir una constelación extensa de proposiciones, interpretaciones, críticas e inflexiones alrededor de un problema-protagonista.

No se trata de hacer una etnografía del laboratorio o de entender el campo científico como un campo de batalla entre ortodoxia y heterodoxia, lo cual los susodichos sociólogos llevaron a cabo con éxito. La introducción de la diacronía, del elemento "tiempo", parece tener por objeto (aunque Oexle no lo mencione explícitamente) problematizar - mejor dicho, conceptualizar críticamente- la noción de "aire de los tiempos" o de "tendencia" que son evocadas tan recurrentemente, al referirse a la producción académico/científica, literaria y artística. ¿Qué necesidades sociales o qué aspectos histórico-coyunturales oculta este "aire de los tiempos"? ¿Qué empuja a la esfera intelectual de una sociedad a insistir sobre ciertos temas/problema? ¿Qué preocupación colectiva subyace tras una "tendencia" académica? He ahí el proyecto de la Problemgeschichte (Oexle, 2011: 808-833).

La Problemgeschichte, tal y como la plantea Oexle, está basada en el precepto metodológico, propuesto inicialmente por Karl Mannheim (1925), de que todo problema está rodeado de un contexto problemático, el cual incluye una variedad de cuestionamientos y proposiciones que efectúan el problema, así como, un conjunto de condiciones sociales entre las que están los sentimientos colectivos, las tendencias históricas y los rasgos culturales-que codeterminan su solubilidad. Consecuentemente, la metodología oexliana exige la 
reconstrucción de dicho contexto problemático (Problemkonstellation) en una perspectiva histórica, con el fin de estudiar a cabalidad el problema-protagonista en sus posibles articulaciones. No se trata, como se expuso anteriormente, de extender la noción estructuralista de "historia de las mentalidades", ni de volver a una "historia de las ideas", más clásica, se trata de ubicarse metodológicamente en una coyuntura entre la Epistemología, la Sociología y la Historia. Se trata de proponer un nuevo campo académico, auténticamente interdisciplinario.

Dicho lo anterior, conviene ahora volver la mirada a la aplicabilidad de la Problemgeschichte para el contexto latinoamericano. Se comienza por decir que la metodología oexliana ofrece un aparato metodológico susceptible de inspirar estudios altamente interesantes para nuestro contexto. Aquí se planteará que, lejos de emprender una reproducción de la temática oexliana, por ejemplo, el historicismo y la crisis de la Realidad/Verdad, para el contexto latinoamericano, sería quizá más provechoso pensar la aplicación de la metodología de la Problemgeschichte para el estudio de problemas-protagonista que han ocupado de forma más tradicional, el mundo intelectual, cultural y artístico de América Latina.

Podrían barajarse varios ejemplos, sin pretender la exhaustividad, tales como, la constelación de cuestionamientos, sentimientos, reflexiones y respuestas que envuelven el problema sobre la Colonia, por ejemplo, lo colonial, lo colonizado y lo colonizable (Stefanell, 2005). Puede pensarse también en la idea misma de la "latinoamericaneidad", que por su extensión, su incidencia y su carácter controversial, constituye el núcleo de una extensa $y$ compleja constelación de problemas (Problemkonstellation). Lo mismo puede decirse con respecto al antiimperialismo (político, académico, filosófico, cultural y popular) (Guajardo, 2007) y su relación con la idea de "dependencia" (Beigel, 2005: 287-326). Este último problema-protagonista es particularmente, apto para ser estudiado utilizando la metodología oexliana, no solo por la gran cantidad de respuestas multidireccionales que genera $y$ las numerosas esferas que toca, sino también por su duración (principios del siglo xix a principios del xxI) - que facilita el aspecto retrospectivo característico de la Problemgeshichte oexliana $-y$ por su relación con la academia, con las políticas gubernamentales y con las ideologías que han atravesado y atraviesan múltiples movimientos sociales.

Lo más importante, empero, no es proporcionar un listado de temas tratados ni de posibles temas a tratar valiéndose de la metodología de la Problemgeschichte oexliana, sino el entender cuáles preguntas en el contexto latinoamericano pueden abordarse plausiblemente, haciendo uso de esta metodología, centrada en encontrar los puntos coyunturales entre la ciencia, la academia, la intelectualidad y su contexto social e histórico. En este sentido, es de vital importancia subrayar que si bien, se propone un marco metodológico evidentemente exógeno a la realidad latinoamericana, en ningún momento se defiende aquí una "importación" de las temáticas explotadas por Oexle, pertinentes a la realidad histórica alemana entre finales del siglo xIx $y$ principios del siglo xx. Los estudios de Oexle sobre el historicismo y sobre la crisis de fe en la posibilidad del conocimiento se suman a un proyecto más amplio que ocupa a este autor desde hace al menos una década, a saber: el estudio de la articulación entre las ciencias básicas, la epistemología, las ciencias sociales ( $y$ de la cultura) y la construcción del totalitarismo en Alemania, concentrándose específicamente, en el período que va desde la unificación del reino (1871) hasta la desarticulación del régimen nacionalsocialista (1944-1945) (Oexle, 2003 y 2004). Dicha empresa no solo es "conveniente" para la aplicación de su metodología, sino que es perfectamente consecuente con su pretensión de examinar críticamente, la articulación entre la academia y su contexto social e histórico.

Ahora bien, si se trata de estudiar la América Latina asumiendo la Problemgeschichte y si se respeta su principio metodológico de contextualización y codeterminación del problema-protagonista, es claro que impera un cambio de temática. Ponderar la aplicabilidad de la Problemgeschichte en un contexto latinoamericano, no es realizable a cabalidad si prima 
el interés por la temática específica y no por la metodología propuesta por Oexle. Tal empresa puede concretarse de forma plausible únicamente, si se piensa este aparato metodológico en función de una problemática que permita, para el contexto latinoamericano, relacionar aspectos de la intelectualidad, la academia, las políticas gubernamentales $y$ los temas de mayor relevancia para la historia propia de la región. Además, si se tiene en cuenta la historia latinoamericana, no puede ignorarse la necesidad de pensar en la articulación entre los problemas-protagonista $y$ los movimientos sociales reivindicativos $y$ de construcción de la autonomía.

En este sentido y para terminar, es quizá pertinente presentar un posible proyecto de investigación, que por su alcance, bien puede abrigar un análisis realizado a partir de la metodología oexliana. Este proyecto que apenas está siendo concebido, se centra en el estudio del arbitraje como método alternativo de resolución de conflictos.

El procedimiento de arbitraje, tal y como se conoce actualmente, tiene sus orígenes en la Edad Media. A partir de fines del siglo xII, en regiones de Francia como Borgoña, Marsella y Tolosa; en las repúblicas italianas como Flandes $y$ en Inglaterra, no solo se recurría al arbitraje para resolver conflictos de orden mercantil, sino que este constituía un procedimiento legítimo y ordinario para la resolución de conflictos civiles. Entre los siglos xvI, xVII y xvIII, al tiempo que las estructuras jurídico-estatales se establecían y organizaban con más claridad, el arbitraje fue relegado esencialmente, al comercio internacional, marítimo y terrestre, hasta transformarse prácticamente, en una mox mercatorum (costumbre de los mercantes) a nivel del procedimiento. Sobre todo durante los siglos xix y principios del xx, las afirmaciones de soberanía nacional hicieron de las cortes estatales $y$ de los precarios intentos de corte internacional, las principales entidades de resolución de litigios internacionales, sobre todo en el contexto de guerra constante en que se encontraban Europa y sus aliados. No obstante, el arbitraje comienza a perder su carácter alternativo con respecto al orden jurídico internacional durante la segunda mitad del siglo xx, hasta transformarse hoy en el método más recurrente de resolución de litigios referentes al comercio y a los contratos internacionales.

Si bien la firma de la Convención de Nueva York en $1958^{4}$, dejaba entrever el proyecto de construir una "esfera de derecho" exclusiva para el comercio internacional y para las compañías transnacionales, es hasta la década de 1980 — momento en que las tendencias neoliberales infiltran las esferas más altas de los gobiernos del mundo- que el arbitraje comienza a jugar un papel protagónico en la resolución de conflictos de tipo económico y comercial a nivel internacional.

Para América Latina, el arbitraje comercial no constituye algo enteramente novedoso. Este ha sido parte en alguna medida de la política exterior estadounidense desde principios del siglo xx. Para 1902, por ejemplo, el conflicto alrededor del bloqueo naval de Venezuela por el gobierno de Theodore Roosevelt, fue resuelto por medio de un arbitraje internacional (Rodríguez, 1977: 277). No obstante, el arbitraje no llega a constituir una parte esencial de la política internacional de los países latinoamericanos hasta luego de la década de 1990.

Un proyecto que estudie el rol del arbitraje internacional en la historia de América Latina puede (o debe), en su vastedad, plantearse la pregunta por la intelligentsia detrás de las operaciones jurídicas y políticas que han permitido instalar este procedimiento en la gran mayoría de códigos comerciales del continente o que lo han transformado en parte esencial de su política internacional. Es en este sentido que la metodología propuesta por Oexle puede resultar de utilidad. Si se retoman los tres pasos fundamentales que propone la metodología oexliana, el orden de la investigación podría eventualmente presentar el siguiente aspecto:

1) Identificación y estudio del problemaprotagonista: en primera instancia, debe plantearse la interrogante referente a la reflexión jurídico-comercial que separa el

4 "Convención para el reconocimiento y aplicación de laudos arbitrales" de la United Nations Commission on International Trade Law, New York, 1958 (ratificada en 1959). 
arbitraje internacional de las formas clásicas de imperialismo durante la primera mitad del siglo xx.

Enseguida, debe estudiarse el nuevo impulso que se da al arbitraje internacional por medio de la vuelta analítica a la Lex Mercatoria, por parte de los intelectuales de la Escuela de Dijon (Goldman, 1964 y Kahn, 1961) y los oxfordianos en las décadas de 1960-1970. A continuación, esta discusión debe trasladarse al contexto estadounidense $y$ al contexto latinoamericano, por medio del estudio de las redes intelectuales. Es de principal interés entender la transmisión de ideas en los círculos universitarios desde Europa y Estados Unidos hasta América Latina. En el contexto anglosajón, por ejemplo, la fundación de la International School of Arbitration, de la Queen's Mary University de Londres, a principios de la década de los 80, marca un hito para la reflexión sobre el arbitraje a nivel universitario. No obstante, en Francia, el Centre de Commerce International (ccI) había jugado un papel importante en la internacionalización del debate jurídico sobre el arbitraje internacional.

El estudio de la reflexión sobre el arbitraje $y$ de la transmisión de ideas debe evidentemente, trascender este minúsculo borrador, que se presenta con el único fin de ilustrar una futura investigación: los casos de México y de Argentina son bastante ilustrativos en lo referente a la transmisión de ideas $y$ a su discusión en el seno de la intelectualidad nacional.

Es en 1971 que México ratifica la Convención de Nueva York, para luego, transformarse en un centro importante de discusión de los métodos arbitrales. Para 1975, el Distrito Federal (DF) era anfitrión del v Congreso Mundial del cci y en 1978, ratifica el Tratado de Arbitraje Internacional de Panamá. El trabajo de los juristas mexicanos (empleados del Estado y miembros de bufetes privados) en pos de legitimar el arbitraje como práctica gubernamental, continúa durante los años 80 , hasta que en 1993, se adopta el modelo de ley para el arbitraje propuesto por la United Nations Commission on International Trade Law (uncirraL). Para la reconstrucción del problema-protagonista resulta esencial la comprensión de las ideas y los métodos utilizados por los juristas latinoamericanos para apoyar y promover el arbitraje, así como comprender su origen y su matriz intelectual. En el caso de los juristas mexicanos, un primer abordaje mostraría que la mayoría fueron estudiantes de la Escuela de Chicago.

El camino argentino es un tanto distinto $y$ está sin duda marcado por la presencia de uno de los principales promotores intelectuales del arbitraje en América Latina, Horacio Grigera Naón (1980, 1989 y 2005), profesor de la Universidad de Harvard, quien desde principios de su carrera, estuvo en contacto con las ideas francesas sobre el arbitraje, habiendo realizado estudios postdoctorales en la Universidad de París II entre 1972 y 1975. El estudio de su trabajo y el de sus pupilos bonaerenses $y$ latinoamericanos, resulta esencial para la comprensión del problema-protagonista.

Debe afirmarse que no puede ser el interés de un estudio de este carácter entender al arbitraje únicamente como un instrumento de dominación, a pesar de que esta concepción resulte sin duda plausible. Es importante comprender primero, el arbitraje como un problema intelectual y jurídico, cuya formulación tiene muy serias implicaciones para la concepción piramidal y kelnesiana del derecho (Kelsen, 1925), así como para la idea de "orden internacional" que esta sostiene, y para la idea del Estado y de lo público. Así las cosas, las ideas neoliberales deben ser puestas en perspectiva, así como, las nuevas concepciones de democracia (o tecnocracia), de orden público y de sistema transnacional. Asimismo, no puede escaparse al estudio de la concepción inicial de un orden jurídico en redes, el cual espeja la noción de un Estado Internacional de posguerra ${ }^{5}$.

2) Reconstrucción de la Problemkonstellation: el segundo paso consistiría en comprender las ramificaciones del problema de la instauración del arbitraje internacional como vía legítima de resolución de conflictos en las políticas internacionales de los países latinoamericanos. En este sentido, es necesario

\footnotetext{
$5 \quad$ Aunque sorprenda, esta noción fue desarrollada en primer lugar en los países bálticos del bloque comunista, en los años 60 (Kahn, 1961: 18).
} 
comprender la oposición que históricamente ha suscitado la implantación del arbitraje en América Latina, especialmente, durante la segunda mitad del siglo xx (Gamboa, 2007: 95-105). No solo interesa estudiar la oposición, reciente o no, mostrada por políticos $y$ movimientos sociales, sino la respuesta intelectual de fondo que justifica la reticencia de las cortes, congresos y consejos de Estado a aceptar el arbitraje, sobre todo en lo relativo a los conceptos de soberanía, orden y bien público, autonomía, injerencia y tamaño del Estado, etc.

La contradicción entre los juristas kelnesianos y los llamados "postkelnesianos", así como, la contradicción entre economistas keynesianos y neoliberales, es de total relevancia para la comprensión de la constelación de problemas. No deben dejarse de lado, de acuerdo al carácter interdisciplinario de la metodología en cuestión, los argumentos de otros sectores como: biólogos, profesionales de los estudios ambientales, científicos sociales, filósofos, etc.

3) Análisis de la dinámica de la Problemkonstellation en un cuadro temporal: como se ha repetido en numerosas ocasiones, la dinámica de la constelación de problemas debe inscribirse en un cuadro temporal. En este caso, se trata del contexto latinoamericano de la segunda mitad del siglo xx. Por lo cual, es necesario entender no solo la transformación y reformulación de las élites latinoamericanas luego de 1960-1970, sino entender los nuevos (y viejos) puntos de discordia al interior de la clase política y sobre todo, identificar los principales elementos que están en juego en la dinámica social $y$ que el arbitraje y el orden que lo enmarca pueden afectar (recursos naturales, tierra, instituciones del Estado, privatizaciones, corrupción, inequidad, etc.). Solo entendiendo este contexto puede comprenderse la compleja dinámica al interior de la constelación de problemas.

El análisis contextual permitiría entender mejor la polarización a lo interno de América Latina con respecto a la aceptación de laudos arbitrales. Por una parte, las negativas de Venezuela, Bolivia, Ecuador, Uruguay, Argentina y Cuba y por otra, la activa recepción y aplicación que se constata en Centroamérica, México, Chile, Brasil y los países del Caribe. Podría preguntarse, por ejemplo, en qué contexto intelectual se desarrollan los argumentos que se utilizaron en los años 80 y 90 para incluir el arbitraje como parte de las cláusulas básicas que integran el acuerdo que dio origen al Mercosur (Ratto, 2007: 25) y cómo, diez años después, ese contexto se transforma radicalmente. De igual importancia es comprender el debate actual que defiende o condena la multiplicación de centros de arbitraje en América Latina y qué aportan a dicho debate los árbitros latinoamericanos, quienes han proliferado en las dos últimas décadas. Los límites del arbitraje y la compleja relación entre el laudo arbitral y el orden jurídico nacional, que se discuten desde hace algunos años, constituirían una parte central de este estudio.

Valga decir, que hasta el momento en que finalizó la redacción de este artículo, su autor no conocía ningún trabajo que emplee la metodología en cuestión para la realización de una investigación retrospectiva ${ }^{6}$. No obstante, se espera haber demostrado la pertinencia y la utilidad de la Problemgeschichte para la investigación histórica, epistemológica, cultural y social en América Latina, así como, se espera que este artículo se sume a los esfuerzos de transmisión y difusión de la metodología oexliana con el fin de suscitar las investigaciones con el carácter mencionado y estimular nuevos puntos de vista que ayuden a dinamizar la academia latinoamericana de hoy.

\section{BIBLIOGRAFÍA}

\section{LIBROS}

Ariès, Philippe. "L'histoire des mentalités". La nouvelle histoire. Jacques Le Goff (ed.). París. PuF, 1978: 167-182.

$6 \quad$ Salvo el trabajo de Moctezuma Franco (2004) anteriormente citado, que parece otorgar igual importancia a la aplicación de la metodología que a la translocalización de la temática oexliana. Cabe mencionar el interesante trabajo de Kozel sobre el barroco americano (2008), aunque el vínculo metodológico con el trabajo de Oexle es únicamente incidental. 
Beigel, Fernanda. Vida, muerte y resurrección de las teorías de la dependencia. Crítica y teoría en el pensamiento social latinoamericano. Buenos Aires. CLACSO, 2006: 287-326.

Bohr, Niels."Zum Komplementaritätsprinzip”. Collected Papers of Niels Bohr 4. Amsterdam: De Boek, 1984.

Bourdieu, Pierre. Sciences de la science et réflexivité. París: Raisons d'agir, 2001.

Brunner, Otto. Land und herrschaft. Badenbei-Wien, 1939.

Deleuze, Gilles. Empirisme et subjectivité. París: PUF, 1953.

Dilthey, Wilhelm. "Einleitung in die Geisteswissenschaften. Versuch einer Grundlegung für das Studium der Gesellschaft und Geschichte". Gesammelte Shcriften 1. Göttingen: Vandenhoeck en Ruprecht, 2006.

Fleck, Ludwig. "Zur krise der wirklichkeit". Erfahrung und Tatsache. Gesammelte aufsätze. Frankfurt am Main: Suhrkamp, 1983.

Gamboa Morales, Nicolás. La inmunidad soberana de jurisdicción en el arbitraje comercial internacional. Evolución y actualidad. Bogotá: Universidad del Rosario, 2007.

Hänel, Michael. "Problemsgeschichte als forschung: die erbschaft des neukantianismus". Das Problem der problemgeschichte. Göttingen: Wallstein Verlag, 2001.

Hegel, Georg Wilhelm. Phänomenologie des Geistes. Leipzig: Reinhard Wolff Verlag, 1988.

Hyppolite, Jean. Genèse et structure de la phénoménologie de l'esprit de Hegel. París: Aubien, 1967.

Jung, Carl. Die Seinsgebundenheit des denkens. Frankfurt am Main: Suhrkamp, 2007.

Kahn, Philippe. La vente commerciale internationale. París: Sirey, 1961.

Kant, Immanuel. El conflicto de las facultades. Roberto Aramayo (ed.). Madrid: Alianza, 2002.

Kantorowicz, Ernst. Der kaiser friedrich der zweite. Münich: Klett-Cotta, 1999.
Kelsen, Hans. Allgemeines Staatslehre. Berlín, 1992.

Koselleck, Reinhart. "Moderne sozialgeschichte und historische zeiten". Theorie der modernen Geshichtsschreibung. Pietro Rossi (ed.). Düsseldorf, 1987.

Kuhn, Thomas. The structures of scientific revolutions. 3era. ed. Chicago, 1996.

Latour, Bruno y Woolgar, Steve. Laboratory life. The social construction of scientific facts. Londres: Sage, 1979.

Laube, Reinhard. Karl Mannheim und die krise des historismus. Göttingen: GUH, 2004.

Lehmann, Hans y Oexle, Otto Gerhard (eds.). Kulturwissenschaft und nationalsozialismus 2. Göttingen: GUH, 2004.

Mannheim, Karl. "Das Problem einer Soziologie des Wissens". Archiv für Sozialwissenschaft und Sozialpolitik 53 (3). 1924: 577-625.

Mannheim, Karl. Eine soziologische theorie der kultur. Frankfurt am Main, 1981.

Meinecke, Friedrich. Die entstehung des historismus. München, 1936.

Moctezuma Franco, Abraham. La historiografía en disputa (México 1940). México: Benemérita Universidad Autónoma de Puebla, 2004.

Nietzsche, Friedrich. Vom Nutzen und Nachteil der Historie für das Leben. Manchen, 1988.

Oexle, Otto Gerhard. Das Problem der Problemgeschichte. Göttingen: Wallstein Verlag, 2001a.

Oexle, Otto Gerhard. L'historisme en débat. De Nietzsche à Kantorowicz. París: PuF, $2001 \mathrm{~b}$

Oexle, Otto Gerhard. Hahn, Heisenberg und die Andere. Berlín, 2003.

Oexle, Otto Gerhard (ed.). Krise des Histoismus, Krise der Wirklichkeit. Göttingen: GuH, 2007.

Oexle, Otto Gerhard. "Begriffsgeschichte -eine noch nicht begriffene Geschichte". Die Wirklichkeit und das Wesen. Göttingen. Vandehoeck y Ruprecht, 2011: 808-833.

Rodríguez Campos, Manuel. Venezuela 1902, la crisis fiscal y el bloqueo. Caracas: Ediciones de la Facultad de 
Humanidades y Educación, Universidad Central de Venezuela, 1977.

Simmel, Georg. Das Wesen der Materie nach Kants Physischer Monadologie. Frankfurt am Main: Suhrkamp, 1992a.

Simmel, Georg. Soziologie. Frankfurt am Main: Suhrkamp, 1992b.

Simmel, Georg. Das Wesen nach Kants physischer Monadologie. Frankfurt am Main: Suhrkamp, 1992c.

Simmel, Georg. "Die Kreuzung sozialer Kreise". Untersuchungen über die Formen der Vergesellschaft. Frankfurt am Main. Suhrkamp, 1992d: 456-511.

Stallmach, Joseph. Dynamis und Energeia: Untersuchungen am Werk des Aristoteles zur Problegmeschichte von Möglichkeit und Wirklichkeit. Meisenheim am Glan: uJv, 1959.

Troeltsch, Ernst. "Die Krisis des Historismus". Die Neue Rundschau 23. 1922: 572-590.

Weber, Max. "Die 'Objektivität' sozialwissenschaftlicher und sozialpolitischer Erkenntnis". Gesammelte Aufsätze zur Wissenschaftslehre. Tübingen. Јсв Mohr, 2003: 146-214.

Woolgar, Steve (ed.). Knowledge and reflexivity: new frontiers in the sociology of knowledge. Londres: Sage, 1988.

ARTÍ́CULOS

Goldmann, Berthold. "Frontières du droit et Lex Mercatoria". Archives de Philosophie du Droit. París, 1964: 177-192.

Grigera Naón, Horacio. "La technique de l'arbitrage comme procédure de révision des contrats". Derecho Comparado 4. Madrid, 1980: 96-148.

Grigera Naón, Horacio. "Arbitration in Latin America: overcoming traditional hostility". Arbitration International 5. Alphen aan den Rijn, 1989: 137-172.

Grigera Naón, Horacio. "Arbitration and Latin America: progress and setbacks". Arbitration International 21 (2). Alphen aan den Rijn, 2005: 127-176 .

Guajardo Soto, Guillermo. "Remozando el nacionalismo y el antiimperialismo latinoamericano". Enfoques 7. Universidad Central de Chile, 2007: 229252.

Heisenberg, Werner. "Über den anschaulichen Inhalt der quantentheoretischen Kinematik und Mechanik". Zeitschrift für Physik 43 (3). Heidelberg, 1927: 172198.

Kozel, Andrés. "El barroco americano y crítica de la modernidad burguesa". Repositorio de la Facultad de Filosofía y Letras. Universidad Autónoma de México, 2008: 163-177.

Mannheim, Karl. "Das Problem einer Soziologie des Wissens". Archiv für Sozialwissenschaft und Sozialpolitik (53) 3. 1925: 577-625.

Ratto, María Celeste. "El proceso de Institution Building del mercosur. Análisis de tratados y acuerdos regionales". Documentos de Jóvenes Investigadores 7. Instituto de Investigaciones Gino Germani UBA, 2007: 1-75.

Stefanell, Alexander. "Civilización/Barbarie en la ciudad letrada de José Rodó y doña Soledad Acosta de Samper: dos ensayistas latinoamericanos del siglo XIX". Historia Caribe 10. Colombia: Universidad del Atlántico, 2005: 111-122.

Fecha de entrega: 16/10/2012 Fecha de aprobación: 22/04/2013 
